\title{
Poroelastic Theory Applied to the Adsorption-Induced Deformation of Vitreous Silica
}

\author{
Benoit Coasne, ${ }^{*, \dagger, \ddagger, \mathbb{I l}}$ Coralie Weigel, ${ }^{\S}, \|$ Alain Polian, ${ }^{\perp}$ Mathieu Kint, ${ }^{\S}, \|$ Jérome Rouquette, ${ }^{\dagger}$ \\ Julien Haines, ${ }^{\dagger}$ Marie Foret, ${ }^{\S}, \|$ René Vacher, ${ }^{\S, \|}$ and Benoit Rufflé*,, ,\| \\ ${ }^{\dagger}$ Institut Charles Gerhardt Montpellier, CNRS/Université Montpellier 2/ENSCM/UMR 5253, F-34095 Montpellier, France

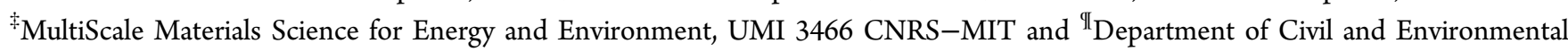 \\ Engineering, Massachusetts Institute of Technology, Cambridge, Massachusetts 02139, United States \\ ${ }^{\S}$ Laboratoire Charles Coulomb UMR 5221, Université Montpellier 2, F-34095 Montpellier, France \\ "Laboratoire Charles Coulomb UMR 5221, CNRS, F-34095 Montpellier, France \\ ${ }^{\perp}$ IMPMC-CNRS UMR 7590, Université Pierre et Marie Curie - Paris 6, B115, 4 pl. Jussieu, F-75252 Paris Cedex 05, France
}

\section{Supporting Information}

\begin{abstract}
When vitreous silica is submitted to high pressures under a helium atmosphere, the change in volume observed is much smaller than expected from its elastic properties. It results from helium penetration into the interstitial free volume of the glass network. We present here the results of concurrent spectroscopic experiments using either helium or neon and molecular simulations relating the amount of gas adsorbed to the strain of the network. We show
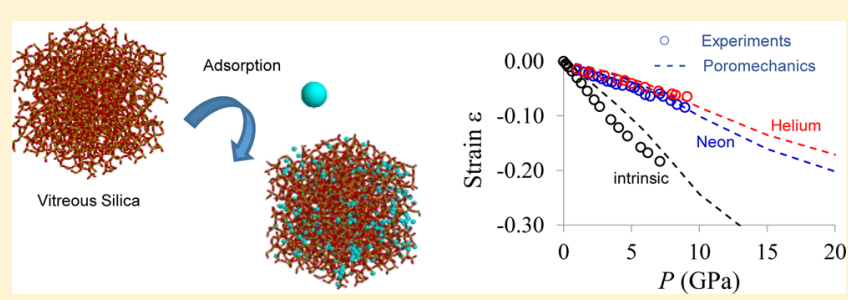
that a generalized poromechanical approach, describing the elastic properties of microporous materials upon adsorption, can be applied successfully to silica glass in which the free volume exists only at the subnanometer scale. In that picture, the adsorptioninduced deformation accounts for the small apparent compressibility of silica observed in experiments.
\end{abstract}

\section{INTRODUCTION}

Gas transport in amorphous materials has been the subject of extensive studies over the past 40 years. Pioneering experiments on noble gas solubility in vitreous silica were conducted up to 0.13 GPa by Shelby. ${ }^{1,2}$ The pressure dependence of $\mathrm{He}$ and $\mathrm{Ne}$ solubilities suggested that the process could be explained by a statistical thermodynamical approach, that is, Langmuir adsorption. In this picture, noble gas solubility is described as a simple physical adsorption process with no chemical bonding to the rigid network. A maximum concentration of $\mathrm{He}$ and $\mathrm{Ne}$ atoms was then estimated to be 0.1 and $0.06 \mathrm{~mol}$ per mole of $\mathrm{SiO}_{2}$, respectively. It was argued that these maximal concentrations represent the tail of a log-normal probability distribution function of interstitial voids with a diameter of around $0.2 \mathrm{~nm}$ and a width of about $0.1 \mathrm{~nm},{ }^{3}$ a result confirmed by computer-generated models. ${ }^{4}$

Recent high-pressure work is producing a new momentum to the field. ${ }^{5-7}$ Below $10 \mathrm{GPa}$, hydrostatic pressures $P$ are obtained in diamond-anvil cells using a soft pressure transmitting medium such as a methanol-ethanol mixture (ME4:1). ${ }^{8}$ When such experiments are performed in the GPa pressure range for vitreous silica, the volume change of the sample agrees with that expected from its bulk modulus $K \simeq$ 35-40 GPa. ${ }^{7,9-13}$ The latter can be obtained by measuring the velocity of both longitudinal $\left(v_{\mathrm{L}}\right)$ and transverse $\left(v_{\mathrm{T}}\right)$ acoustic waves, $K=\rho\left(v_{\mathrm{L}}^{2}-(4 / 3) v_{\mathrm{T}}^{2}\right)$, where $\rho$ is the mass density of the sample. On the other hand, the compressibility of vitreous silica inferred from the volumetric strain is strongly reduced when $\mathrm{He}$ is used as a pressurizing medium, ${ }^{5,6}$ leading to the apparent bulk modulus $K_{\text {app }} \simeq 110-120 \mathrm{GPa}$. Using Brillouin light scattering, it is shown in ref 7 that $K$ is only weakly affected by the presence of $\mathrm{He}, K \simeq 35-50 \mathrm{GPa}$, demonstrating that the much smaller apparent compressibility is not due to a stiffening of the network. The large difference between $K_{\text {app }}$ and $K$ indicates that silica pressurized under He behaves as an open system. In that case, the static measurement underestimates the compressibility of the network by a term due to the gas charging the sample.

The complex interplay between fluid adsorption, mechanical response, and structural modifications in glasses remains fundamental yet presently unresolved. It relates to the open and flexible structure of the glass, which allows gas atoms to distend the network, in the same way as a porous material would deform upon fluid adsorption. ${ }^{14-20}$ Though glasses are not characterized by an open porosity, it is expected that thermal motion opens the interstitial voids to gas atom diffusion. Hence, an adapted poromechanical description of adsorption-induced deformation of glasses makes sense. Such general poroelastic constitutive equations, which were revisited only recently, ${ }^{21}$ offer a very appealing theoretical framework.

Received: September 17, 2014

Revised: November 7, 2014

Published: November 10, 2014 
The present work reports a joint experimental and theoretical study providing a unifying and comprehensive picture of adsorption-induced deformation of bulk amorphous silica. We show that generalized poromechanics, a theory that was initially developed to describe the properties of porous elastic materials upon adsorption, can be extended to dense glass networks exhibiting only interstitial voids.

\section{METHODS}

2.1. Experimental Techniques. Using $\mathrm{Ne}$ as a pressurizing medium, we measured the changes in volume of vitreous silica as a function of fluid pressure up to $9 \mathrm{GPa}$. The silica samples, Suprasil SF300 with less than 1 ppm of $\mathrm{OH}$ content from Heraeus QuartzGlass, Germany, were loaded in a Chervin-type diamond-anvil cell using ruby chips for pressure calibration. ${ }^{22}$ The pressure dependence of the sample volume change was obtained by the measurement of the area of its image recorded with a CCD camera.

We further measured by Brillouin scattering the longitudinal and transverse sound velocities of vitreous silica immersed in $\mathrm{Ne}$ up to $7 \mathrm{GPa}$. Brillouin spectra were obtained with the 514.5 $\mathrm{nm}$ line of a single-frequency argon ion laser. A high-resolution setup $^{23}$ was used in backscattering to precisely measure the longitudinal component. A Sandercock tandem interferometer was used in the platelet geometry to acquire simultaneously the longitudinal and transverse components. The Brillouin frequency shift $\nu_{\mathrm{B}}$ in an isotropic medium is given by

$$
\nu_{\mathrm{B}}=\frac{2 n v}{\lambda_{0}} \sin \frac{\theta}{2}
$$

where $n$ is the refractive index, $v$ the sound velocity of the mode responsible for the scattering, $\lambda_{0}$ the wavelength of the incident radiation in vacuum, and $\theta$ the scattering angle inside the sample. In backscattering, this equation reduces to $\nu_{\mathrm{B}}^{\text {bs }}=2 n v / \lambda_{0}$. In the symmetric platelet geometry, ${ }^{24}$ one gets $\nu_{\mathrm{B}}^{\mathrm{p}}=\left(2 v / \lambda_{0}\right)$ $\sin \left(\theta_{\text {ext }} / 2\right)$, where $\theta_{\text {ext }}$ is the angle between the incident and scattered beams outside the sample. $\nu_{\mathrm{B}}^{\mathrm{p}}$ does not depend on the refractive index of the sample. This gives a way to extract $n$ from measurements of the same mode in both geometries

$$
n=\frac{\nu_{\mathrm{B}}^{\mathrm{bs}}}{\nu_{\mathrm{B}}^{\mathrm{p}}} \sin \frac{\theta_{\mathrm{ext}}}{2}
$$

2.2. Poromechanical Model. Adsorption-induced deformation (swelling or shrinkage) of porous materials can be described in the frame of classical poromechanics. This theory describes the volumetric strain $\epsilon$ and shear strain $e_{i j}$ induced by the adsorption of fluid molecules at a pressure $P .^{14,25}$ For an elastic solid having an intrinsic porosity $\Phi_{0}$ and submitted to small strains, the constitutive equations read

$$
\begin{gathered}
\sigma=K_{0} \epsilon-b P \\
\phi=\Phi-\Phi_{0}=b \epsilon+\frac{P}{N} \\
s_{i j}=2 G_{0} e_{i j}
\end{gathered}
$$

where $K_{0}$ and $G_{0}$ are the constant bulk and shear moduli while $\sigma$ and $s_{i j}$ are the volume and shear stresses. $\phi$ is the porosity variation because $\Phi$ and $\Phi_{0}$ are the porosities in the presence and in the absence of the adsorbed fluid, respectively. $b$ and $N$, which are referred to as the Biot coefficient and modulus, respectively, describe the coupling between the adsorption of molecules and the deformation of the material. A well-known drawback of eq 3 is that the fluid confined in the porosity of the material is assumed to behave as the bulk fluid, that is, no confinement effect. While this is a reasonable assumption for macroporous solids (pores larger than $50 \mathrm{~nm}$ ), this does not hold for smaller pores such as mesopores $(2-50 \mathrm{~nm})$ and micropores $(<2 \mathrm{~nm})$. Of course, this approximation is even less justified for the interstitial cavities $(<0.4 \mathrm{~nm})$ existing in bulk glasses which arise from inherent density fluctuations. The theory has been recently revisited to include physical adsorption phenomena in microporous solids without any assumption on the state of the confined fluid and on the pore geometry. ${ }^{21}$ The approach is based on energy conservation and follows from first-principles of thermodynamics. The constitutive equation, valid for an isotropic linear elastic porous solid under the hypothesis of small deformation, relates $\sigma$ to $\epsilon$ and to the number of fluid atoms per unit volume of the undeformed solid $n(\epsilon, P)$, the latter appearing naturally as a state variable of the system

$$
\sigma=K_{0} \epsilon-\frac{\partial}{\partial \epsilon}\left[\int_{0}^{P} n(\epsilon, p) V_{\mathrm{b}}(p) \mathrm{d} p\right]_{P}
$$

Here, $V_{\mathrm{b}}(P)$ is the molecular volume of the bulk fluid. Equation 4 has been shown to be consistent with classical poromechanics when the incorporated fluid is in its bulk state. ${ }^{21}$ Note that eq 4 describes the thermodynamic equilibrium state of the system after all memory effects have taken place.

In standard high-pressure experiments in diamond-anvil cells, the sample is immersed into the pressurizing fluid. The confining stress applied to the solid is thus equal to the opposite of the bulk fluid pressure, $\sigma=-P$, and no wall or membrane separates the sample from the bulk fluid. The solid is free to shrink or to swell. This is the so-called unjacketed compression test in the field of poromechanics. In such experiments, the pressure $P$ of the bulk fluid drives both the amount of adsorbed atoms $n^{u}(P)$ and the volumetric strain $\epsilon^{u}(P)$ of the solid, and eq 4 yields a relation that $\epsilon^{u}(P)$ must verify

$$
\epsilon^{u}(P)=-\frac{P}{K_{0}}+\left.\frac{1}{K_{0}} \int_{0}^{P} \frac{\partial n(\epsilon, p)}{\partial \epsilon}\right|_{\epsilon=\epsilon^{u}} V_{\mathrm{b}}(p) \mathrm{d} p
$$

Equation 5 shows that the knowledge of the adsorption isotherms $n(\epsilon, P)$ is sufficient to predict the pressure dependence of $\epsilon^{u}(P)$ and concomitantly $n^{u}(P)$. The mechanical deformations can thus be predicted without having to simulate directly the material deformation induced upon adsorption at a given $P$. This approach is consistent with other thermodynamical treatments of adsorption-induced flexibility in metalorganic frameworks and swelling of coals ${ }^{16,26}$ and has been recently extended to take into account the anisotropy of material properties. $^{27}$

In the specific case of coal swelling induced by $\mathrm{CO}_{2}$ or $\mathrm{CH}_{4}$ molecules, molecular simulations have shown that the adsorbed amount increases almost linearly with the volumetric strain, ${ }^{21}$ $n(\epsilon, P) \simeq n^{0}(P)(1+C(P) \epsilon)$, where $n^{0}(P)$ is the amount of adsorbed molecules at zero strain and $C(P)=\partial n(\epsilon, P) / \partial \epsilon$. According to eq 5 , the pressure dependence of the strain in unjacketed conditions $\epsilon^{u}(P)$ is then directly related to $n^{0}(P)$ and $C(P)$ extracted from either measured or simulated adsorption isotherms 


$$
\epsilon^{u}(P)=-\frac{P}{K_{0}}+\frac{1}{K_{0}} \int_{0}^{P} n^{0}(p) C(p) V_{\mathrm{b}}(p) \mathrm{d} p
$$

which in turn gives the adsorbed amount of molecules in unjacketed conditions $n^{u}\left(\epsilon^{u}, P\right)$.

In our analysis of the adsorption-induced deformation of vitreous silica, we used a slightly more refined strategy to calculate $\epsilon^{u}(P)$. First, it is well-documented that in the GPa pressure range, vitreous silica does not behave as a linear elastic solid. ${ }^{7,9-13}$ The bulk modulus first decreases from $K_{0} \simeq 36 \mathrm{GPa}$ at ambient pressure to $K \simeq 30 \mathrm{GPa}$ at around $P=2 \mathrm{GPa}$ and then increases rapidly with increasing $P$, reaching $K \simeq 43 \mathrm{GPa}$ at $P=6 \mathrm{GPa}$. A similar behavior ${ }^{28}$ is observed in the model silica that we used, which is described in the following section. $K$ decreases from $K_{0} \simeq 56 \mathrm{GPa}$ at ambient pressure to $K \simeq 30$ $\mathrm{GPa}$ at around $P=8 \mathrm{GPa}$ and then increases at higher $P$. As an attempt to take into account the pressure dependence of $K$, one can use the secant bulk modulus $K^{s}(P)=-P / \epsilon$ of the solid in the absence of any adsorbed fluid in eq $5 . K^{s}(P)$ indeed relates the bulk fluid pressure $P$ exerted on the sample to the volume deformation that would be observed without adsorption. $K^{S}(P)$ was derived from ref 28 . Such an attempt was made to test whether possible stiffening of the sample could change the predictions of the poromechanical model. In fact, rigorously, the model is only valid when $K_{0}$ is used in eq 6 . Using $K_{0}$ or $K^{s}(P)$ leads to very similar results as $K_{0} \simeq K^{s}(P)$ in the pressure range considered in the present work, as shown in Figure 1.

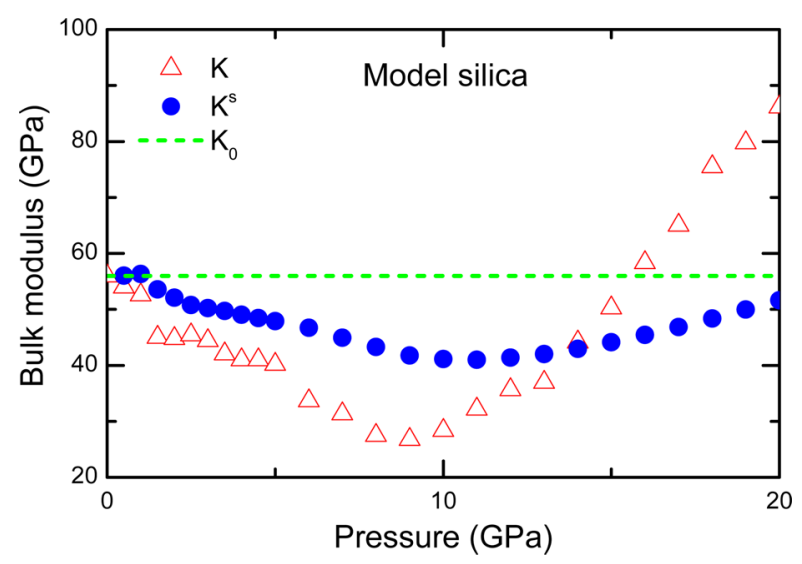

Figure 1. Pressure dependence of the bulk modulus of model silica (red triangles). ${ }^{28}$ Blue dots are the secant bulk modulus used in the poromechanical model, and the green dashed line shows the $P=0$ value $K_{0}$.

Second, the simulated adsorption isotherms are not linear with strain but increase almost quadratically. An iterative procedure was then developed to calculate $\epsilon^{u}(P)$

$$
\epsilon_{i+1}^{u}(P)=-\frac{P}{K^{s}}+\left.\frac{1}{K^{s}} \int_{0}^{P} \frac{\partial n(\epsilon, p)}{\partial \epsilon}\right|_{\epsilon=\epsilon_{i}^{u}} V_{\mathrm{b}}(p) \mathrm{d} p
$$

The unique solution for $\epsilon^{u}(P)$ is obtained after a couple of steps, using either $\epsilon_{0}(P)=-P / K_{0}$ or $\epsilon_{0}(P)=0$ as initial values. The corresponding adsorbed amount $n^{u}\left(\epsilon^{u}, P\right)$ at the pressure $P$ is then straightforwardly derived from $\epsilon^{u}(P)$ following the adsorption isotherms.

2.3. Molecular Simulations. Adsorption at room temperature of $\mathrm{He}$ and $\mathrm{Ne}$ in dense silica was simulated for different volumetric strains $\epsilon$ by means of Grand Canonical Monte Carlo
(GCMC) simulations. In this technique, the system (silica and fluid) is in equilibrium with a fictitious reservoir imposing its temperature $T$ and chemical potential $\mu$ to the fluid. The calculations were performed for a cubic sample of bulk amorphous silica having a length of $3.6 \mathrm{~nm}$. Periodic boundary conditions were used along the $x, y$, and $z$ directions in order to avoid finite size effects. Following previous work on highpressure adsorption in porous materials, ${ }^{29} \mu(T, P)$ for He and Ne was estimated from the fugacity $f=\exp \left(\mu / k_{\mathrm{B}} T\right)$ using the virial equation of state. Such GCMC simulations, which consist of generating atomic configurations and accepting/rejecting them on the basis of the Boltzmann factor in the Grand Canonical ensemble, do not allow probing the dynamics of the system (even if the equivalence, i.e. ergodicity principle, between molecular dynamics and GCMC was demonstrated in the case of adsorption in porous media ${ }^{30}$ ). While probing the dynamics of amorphous silica subjected to high-pressure adsorption would provide interesting insights into the corresponding relaxation dynamics, it is out of the scope of the present paper.

The force field developed by ref 31 was used to describe silica. In this model, the $\mathrm{O}$ and $\mathrm{Si}$ atoms, carrying partial charges, interact through a Coulombic term and a repulsion/ dispersion term described using a Buckingham potential, while Lennard-Jones (LJ) potentials were used for $\mathrm{He}$ and Ne. Unlike atom LJ parameters between the noble gas atoms and silica were obtained from the like-atom parameters using the Lorentz-Berthelot mixing rules (like-atom LJ parameters for silica are taken from ref 32 while those for He were from ref 33 and those for Ne were from ref 34 ). The LJ parameters, $\sigma_{\mathrm{LJ}}$ and $\epsilon_{\mathrm{LJ}}$, used to model the atomic interactions are given in Table 1 . The electrostatic interaction was calculated using the Ewald sum to correct for the finite size of the simulation box.

Table 1. LJ Parameters Used in the GCMC Simulations ${ }^{a}$

$\begin{array}{ccccccc}\mathrm{X} & \sigma_{\mathrm{X}-\mathrm{X}} & \sigma_{\mathrm{X}-\mathrm{O}} & \sigma_{\mathrm{X}-\mathrm{Si}} & \epsilon_{\mathrm{X}-\mathrm{X}} & \epsilon_{\mathrm{X}-\mathrm{O}} & \epsilon_{\mathrm{X}-\mathrm{Si}} \\ \mathrm{He} & 0.264 & 0.2897 & 0.32175 & 10.9 & 29.16 & 26.45 \\ \mathrm{Ne} & 0.282 & 0.2987 & 0.33075 & 32.8 & 50.59 & 45.88 \\ { }^{a} \sigma \text { is in } \mathrm{nm} \text {, and } \epsilon \text { is in } \mathrm{kJ} / \mathrm{mol} \text {. }\end{array}$

Practically, the adsorption isotherms $n(\epsilon, P)$ are obtained by means of GCMC simulations as varying independently the deformation $\epsilon$ and the bulk fluid pressure $P$ in the GPa range is not an easy task in laboratory experiments. The bulk modulus $K$ of the sample at ambient pressure is estimated using Monte Carlo or molecular dynamics simulations in the isothermalisobaric ensemble (NPT) from the pressure-induced volume variation of the system in the absence of any adsorbed fluid. The value found is similar to the value reported in ref 28 , where the same effective potential was used to simulate vitreous silica.

\section{RESULTS AND DISCUSSION}

\subsection{Revealing the Adsorption-Induced Deformation.} The measured volumetric strains of $\mathrm{v}^{-\mathrm{SiO}_{2}}$ immersed in $\mathrm{Ne}$ fluid at pressures below $9 \mathrm{GPa}$ are shown in Figure 2. The results are compared with those obtained previously under $\mathrm{He}$ atmosphere at room temperature. ${ }^{5}$ In the latter case, when a pressure step is applied, the volumetric strain appears instantaneously on the time scale of the experiment. In contrast, under a $\mathrm{Ne}$ atmosphere at room temperature, the equilibrium is reached after a delay that can extend to hours or even days, depending on $P$ and sample sizes. An example of $\mathrm{Ne}$ 


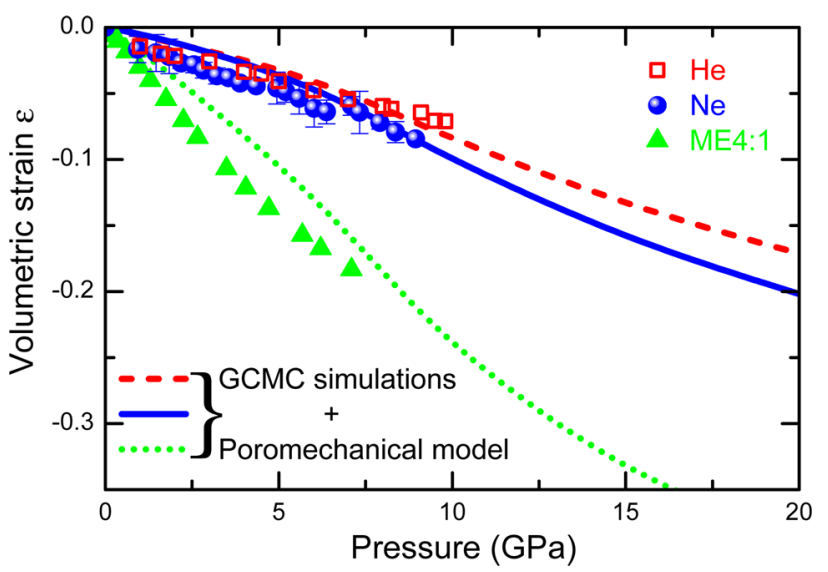

Figure 2. Volumetric strain of $\mathrm{v}-\mathrm{SiO}_{2}$ as a function of the pressure exerted by an adsorbing fluid, $\mathrm{Ne}$ (blue dots), $\mathrm{He}$ (red squares), ${ }^{5}$ and the nonpenetrating fluid ME4:1 (green triangles). ${ }^{7}$ The solid and the dashed lines show the theoretical predictions as discussed in the text for $\mathrm{He}$ and $\mathrm{Ne}$, respectively. The dotted line shows the deformation of the model silica in the absence of adsorption. ${ }^{28}$

adsorption kinetics is illustrated in Figure 3. When a pressure step from 0.2 to $2.1 \mathrm{GPa}$ is applied at time $t=0$, the volume

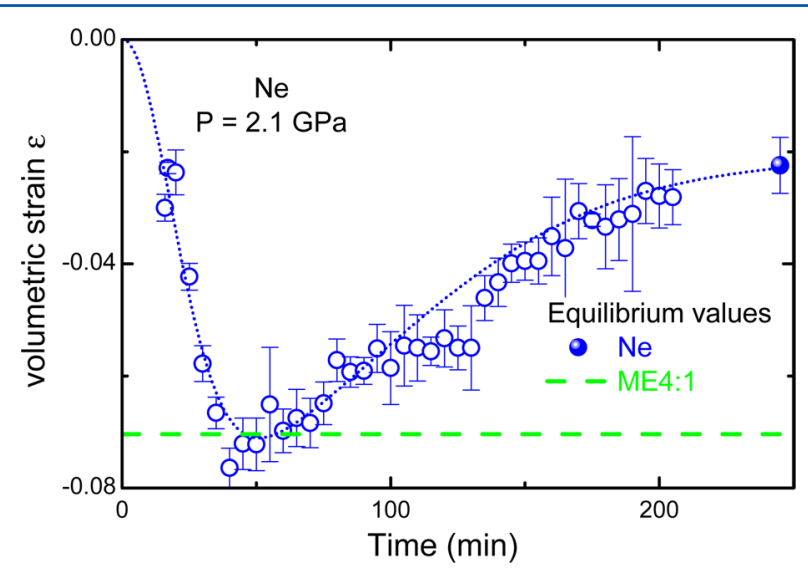

Figure 3. Volumetric strain as a function of time after a pressure step from 0.2 to $2.1 \mathrm{GPa}$ in Ne. The dotted line is a guide for the eye. The equilibrium value is shown by a full symbol. The dashed line marks the expected deformation of silica immersed in the nonpenetrating fluid ME4:1.

first decreases to the value obtained with a nonpenetrating pressurizing fluid $(\epsilon \simeq-0.07)$, showing that $\mathrm{Ne}$ has not appreciably diffused into the network at this stage. At longer times, the volume increases and eventually reaches an equilibrium value. This increase in volume shows that $\mathrm{Ne}$ penetration unexpectedly distends the silica network at constant external pressure. As shown in Figure 2, the equilibrium values of the volumetric strain in the $\mathrm{Ne}$ atmosphere are only slightly smaller than those found with $\mathrm{He},{ }^{5}$ indicating that the penetration of $\mathrm{Ne}$ produces almost the same effect as that of He. In both cases, the change in volume is much smaller than the deformation obtained when vitreous silica is pressurized using the nonpenetrating fluid ME4:1.

Direct measurement of the amount of gas entering a solid upon high pressures is a very difficult task. We attempted to obtain this information in situ from the refractive index of the $\mathrm{He}$ or $\mathrm{Ne}$ stuffed samples. We have used an effective medium approximation to estimate the mean polarizability of the composite. We applied the Clausius-Mossotti relation, $3 \epsilon_{0}\left[\left(n^{2}\right.\right.$ $\left.-1) /\left(n^{2}+2\right)\right]=N_{\mathrm{SiO}_{2}} \alpha_{\mathrm{SiO}_{2}}+N_{\mathrm{f}} \alpha_{\mathrm{f}}$, where $N_{\mathrm{f}}$ is the atomic density of adsorbed fluid atoms, whereas $\mathrm{N}_{\mathrm{SiO}_{2}}$ is the atomic density of the $\mathrm{SiO}_{2}$ molecules, extracted from the volumetric strain measurements. $\alpha_{\mathrm{f}}$ is the polarizability of the fluid, which hardly depends on $P$ in the pressure range investigated in the present work. ${ }^{35,36} \alpha_{\mathrm{SiO}_{2}}$ is the strain-dependent polarizability of the silica network taken from the measurements at the same volumetric strain in a nonpenetrating pressurizing medium. ${ }^{11}$ Figure 4 shows the strain dependence of the ratio $\left(n^{2}-1\right) /\left(n^{2}\right.$

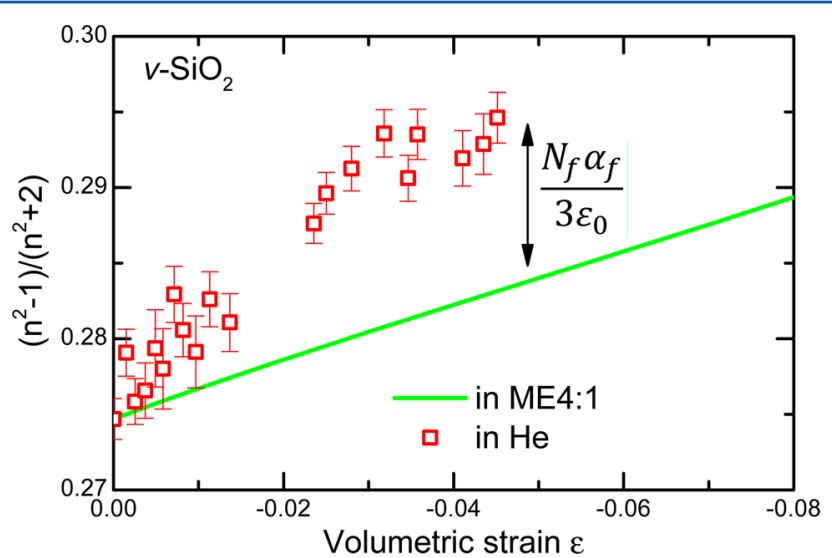

Figure 4. Strain dependence of the ratio $\left(n^{2}-1\right) /\left(n^{2}+2\right)$ for vitreous silica immersed in He (symbols) and in ME4:1 (line $\mathrm{i}^{11,12}$ ). The difference between the two data sets is related to the amount of $\mathrm{He}$ adsorbed in the silica network.

+ 2) for vitreous silica immersed in He and in ME4:1. In the former case, the error bars mainly reflect the uncertainty on the exact symmetric platelet geometry affecting the internal scattering angle $\theta$ in Brillouin scattering experiments. The difference between the symbols and the line at a given $\epsilon$ provides an estimate of $N_{\mathrm{f}}(\epsilon)$ in the stuffed sample. It must be stressed that $N_{\mathrm{f}}$ can be estimated only when the difference between the two refractive indices is significant, that is, in the high-pressure region. From the measured $N_{\mathrm{f}}(\epsilon)$ and $\epsilon(P)$, $N_{\mathrm{f}}(P)$ can be calculated straightforwardly. In the upper pressure region investigated, we found $N_{\mathrm{f}} \simeq 20 \pm 4$ at $/ \mathrm{nm}^{3}$ in He at a pressure of $P \approx 6 \mathrm{GPa}$ (for comparison, the number density of silica at this $\mathrm{He}$ pressure is $\mathrm{N}_{\mathrm{SiO}_{2}} \approx 23 \mathrm{SiO}_{2} / \mathrm{nm}^{3}$ ). Although a similar variation of the refractive index for silica immersed in $\mathrm{Ne}$ is observed, the higher Ne polarizability results in a lower adsorbed quantity of $\mathrm{Ne}$ atoms of $\simeq 7 \pm 4 \mathrm{at} / \mathrm{nm}^{3}$ in Ne. These values can be compared to those found for $\mathrm{H}_{2}$ adsorbed in vitreous silica, $\simeq 10.4$ at $/ \mathrm{nm}^{3}$ at $P=6 \mathrm{GPa}$, using an ex situ quenching technique. $^{37}$

The equilibrium values of sound velocities of vitreous silica immersed in $\mathrm{Ne}$ up to $7 \mathrm{GPa}$ are shown in Figure 5 (blue dots) together with our previous results obtained in $\mathrm{He}$ (red squares). Sound velocities closely follow those found under He. As discussed in ref 7 for $\mathrm{He}$, the anomalous minimum at $2 \mathrm{GPa}$ in $v_{\mathrm{L}}(P)$ of vitreous silica (green triangles) ${ }^{9-11,13}$ is almost completely suppressed in Ne. This indicates that the gas atoms penetrate into the silica network and first fill the largest interstitial voids, which are mainly responsible for the anomalous softening of the network..$^{6,7,38,39}$ Figure 5 also shows that at a given $P$, the sound velocities in $\mathrm{He}$ or $\mathrm{Ne}$ are only slightly larger than those obtained in the nonpenetrating 


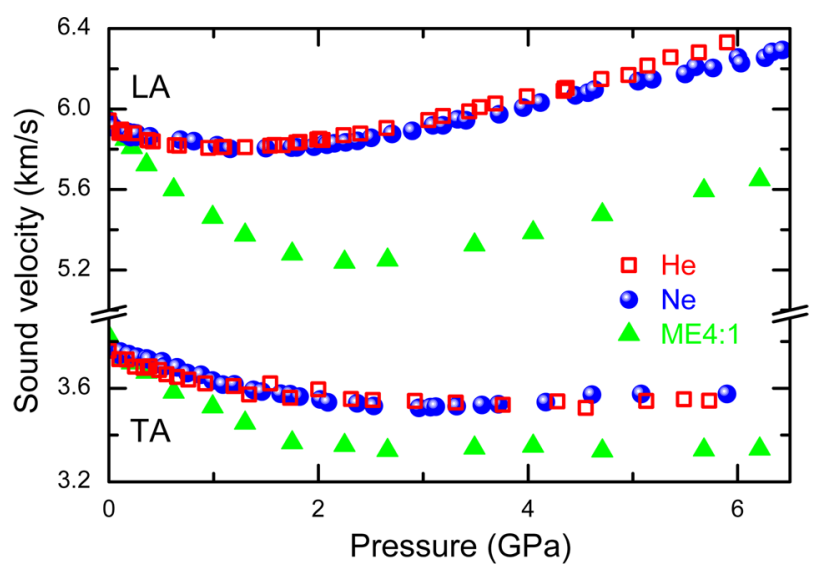

Figure 5. Sound velocities of $\mathrm{v}_{-} \mathrm{SiO}_{2}$ as a function of $P$ immersed in $\mathrm{Ne}$ (blue dots), He (red squares), ${ }^{7}$ and ME4:1 (green triangles). ${ }^{7}$ LA and TA denote the longitudinal and transverse acoustic velocities, respectively.

pressurizing medium ME4:1. This indicates that the much lower apparent compressibility deduced from volume changes shown in Figure 2 is not due to a huge stiffening of the network but rather to an adsorption-induced deformation of the glass network as illustrated by Figure 3. Such a strong effect of the pressurizing medium on the static compressibility, associated with adsorption, is usually observed in materials with open porosity, such as zeolites. ${ }^{29}$ Though glasses are not porous materials, the above-mentioned observations strongly suggest that an adapted poromechanical theory might describe the adsorption-induced phenomena in glasses.

3.2. Poromechanical Description. GCMC simulations in the frame of the above-mentioned generalized poromechanics were performed to probe at the microscopic scale the deformation of vitreous silica upon adsorption. As explained in section 2.3, one first determines the number $n(\epsilon, P)$ of $\mathrm{Ne}$ or $\mathrm{He}$ atoms per unit volume of solid penetrating a realistic model of silica as a function of the volumetric strain and bulk fluid pressure, $\epsilon$ and $P$ being taken as independent external variables. ${ }^{29} n(\epsilon, P)$ is shown in Figure 6, where we report the

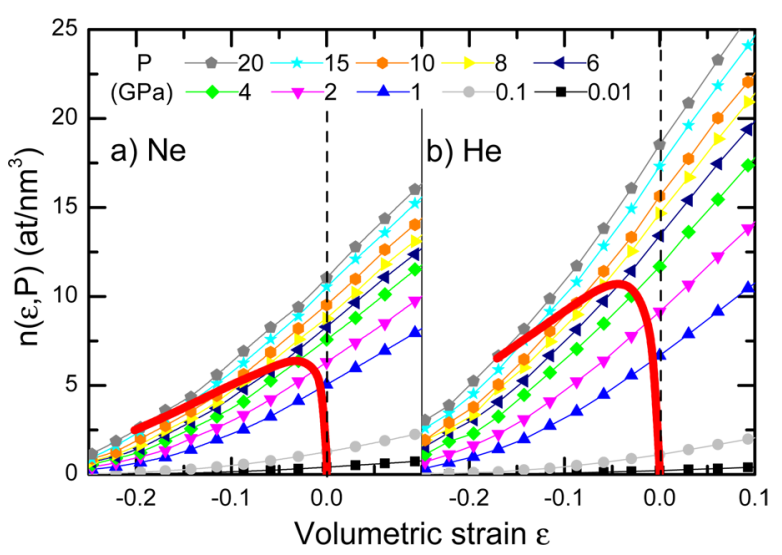

Figure 6. Room-temperature adsorption isotherms in $\mathrm{v}-\mathrm{SiO}_{2}$ from GCMC simulations as a function of the bulk fluid pressure $P$ : $\mathrm{Ne}(\mathrm{a})$, $\mathrm{He}$ (b). The symbols represent the calculated values, whereas the thin lines are guides for the eye. The vertical dashed lines mark the unstrained volume. The bold red lines show the amount of gas atoms adsorbed in silica predicted by the poroelastic constitutive equations in unjacketed conditions $n^{u}\left(\epsilon^{u}, P\right)$. adsorbed amount as a function of $\epsilon$ for different pressures $P$ (we also show in Figure S1 of the Supporting Information the $\mathrm{Ne}$ and $\mathrm{He}$ adsorption isotherms in the silica glass obtained for different strains $\epsilon$ ). For both fluids, the adsorbed amount increases monotonically with increasing volumetric strain and bulk fluid pressure. Given that the molecular density of the model silica is $\sim 21.8 \mathrm{~mol} / \mathrm{nm}^{3}$, the simulated adsorption isotherms show that relatively high $\mathrm{He}$ and $\mathrm{Ne}$ solubilities are reachable. At given $\epsilon$ and $P$, the number of $\mathrm{He}$ atoms adsorbed in the silica network is about 1.5 times that of $\mathrm{Ne}$ atoms, consistent with the larger size of $\mathrm{Ne}$.

We now turn to the predictions of the generalized poromechanical theory for $\epsilon^{u}(P)$ and $n^{u}(P)$. Using eq 7 and the simulated adsorption isotherms of Figure 6, the unique solution for $\epsilon^{u}(P)$ is found. The pressure dependence of $\epsilon^{u}(P)$ predicted by the poroelastic model is shown in Figure 2 as solid and dashed lines for $\mathrm{Ne}$ and $\mathrm{He}$, respectively. The calculated values reproduce rather nicely the experimental volumetric strains. The fact that the theoretical deformation $\epsilon^{u}(P)$ slightly overestimates the experimental data at low $P$ is believed to be mostly due to the larger bulk modulus of the simulated silica. The volumetric strain of the model silica in the absence of adsorption indeed overestimates the experiments, as shown in Figure 2 by the dotted line and the triangles. The above results show that the poromechanical approach provides a framework for a quantitative description of the adsorption-induced deformation in vitreous silica. In particular, in agreement with the experimental data, our calculations show that the silica network expansion induced by $\mathrm{He}$ adsorption is larger than that induced by $\mathrm{Ne}$ adsorption.

The corresponding adsorbed amount $n^{u}\left(\epsilon^{u}, P\right)$ is shown by the bold red curves in Figure 6 to enlighten the method and in Figure $7 \mathrm{~b}$ as a function of $P$. A typical atomic configuration

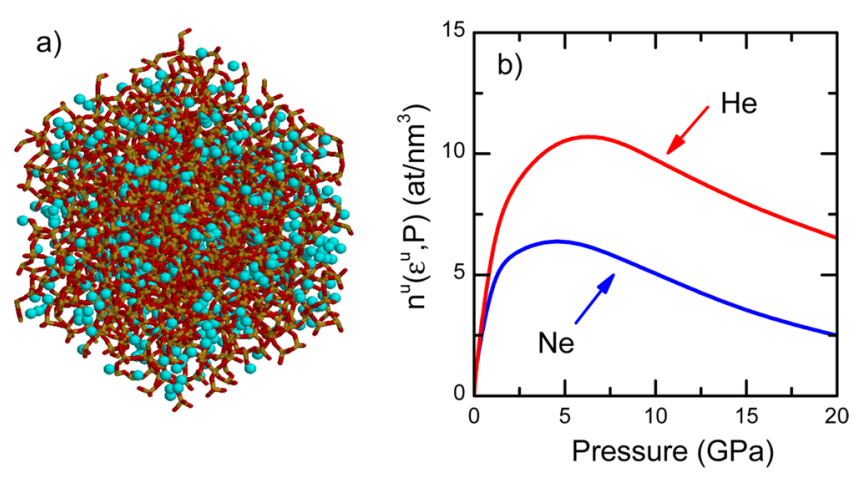

Figure 7. (a) Typical atomic configuration for He adsorbed at room temperature in silica glass at a bulk fluid pressure $P=6 \mathrm{GPa}$ and a fixed volumetric strain $\epsilon=-0.03$. To emphasize the large amount of adsorbed fluid atoms, about $11 \mathrm{at} / \mathrm{nm}^{3}$, only $\mathrm{He}$ atoms are shown (blue spheres). The silica network is shown by sticks that are orange on the $\mathrm{Si}$ side and red on the $\mathrm{O}$ side. The simulation cube is projected along its $[x, y, z]$ diagonal. (b) Pressure dependence of the amount of $\mathrm{Ne}$ and $\mathrm{He}$ atoms adsorbed by $\mathrm{v}-\mathrm{SiO}_{2}$ predicted by the poroelastic model in unjacketed conditions.

close to the red curve for He adsorbed in silica glass at $P=6$ GPa and $\epsilon=-0.03$ is shown in Figure 7a, illustrating the large amount of adsorbed gas atoms. In this case, the $\mathrm{He}$ concentration is about 11 at $/ \mathrm{nm}^{3}$, that is, $0.53 \mathrm{~mol}$ of He per mole of $\mathrm{SiO}_{2}$, in qualitative agreement with the value of $N_{\mathrm{f}}$ estimated from the experiments $\left(20 \pm 4\right.$ at $/ \mathrm{nm}^{3}$ for He and $7 \pm$ $4 \mathrm{at} / \mathrm{nm}^{3}$ for $\left.\mathrm{Ne}\right)$. The amount of $\mathrm{He}$ adsorbed in silica is 
almost twice that of $\mathrm{Ne}$ at the same $P$. In both cases, the predicted $n^{u}(P)$ varies nonmonotonically with $P$. It first increases rapidly to a maximum at around 4 and $6 \mathrm{GPa}$ for $\mathrm{Ne}$ and $\mathrm{He}$, respectively, and then decreases as the mechanical stress exerted by the fluid outside of the sample induces significant volume contraction. We note that a similar pressure dependence has been recently obtained for the solubility of argon in liquid silica using very long molecular dynamics simulations of the complete system equilibrated at fixed $T$ and $P .^{40}$ Alternatively, the adsorption capacity can be quantified by the tangent Biot coefficient, $b^{\tan }(\epsilon, P)=V_{\mathrm{b}}(P)[\partial n(\epsilon, P) / \partial \epsilon]=1$ $-K / K_{\text {app }}$, in the unjacketed case. The tangent Biot coefficients derived from the experiments are $b^{\text {tan }} \simeq 0.7$ for He and $b^{\text {tan }} \simeq$ 0.6 for $\mathrm{Ne}$, in qualitative agreement with the values given by molecular simulations $\left(b^{\tan }=0.83\right.$ and $b^{\tan }=0.74$ for $\mathrm{He}$ and $\mathrm{Ne}$ at $2 \mathrm{GPa}$ ). Interestingly, such high Biot coefficients are usually encountered in porous materials such as limestone or sandstone. ${ }^{41,42}$

\section{CONCLUSION}

The present study shows that the small apparent compressibility of silica under high $\mathrm{He}$ or $\mathrm{Ne}$ pressure is due to an adsorption-induced expansion. It further demonstrates that the elasticity of vitreous silica subjected to noble gas adsorption can be described by generalized poromechanics. This theory for the mechanical properties of microporous solids upon adsorption is applied here to an amorphous solid exhibiting only interstitial voids, opening the way to a broad range of applications. We anticipate that similar conclusions will be reached for other glasses exhibiting a significant amount of interstitial free volume as in the case of $\mathrm{GeO}_{2}$ or $\mathrm{B}_{2} \mathrm{O}_{3}$. The combined use of Monte Carlo simulations and poromechanics provides an efficient alternative to other theoretical approaches ${ }^{16,26,40,43}$ describing the complete system equilibrated in isothermal-isobaric or osmotic ensembles but inherently limited to short time scales and by possible equilibration issues. Poromechanics associated with Monte Carlo simulations thus might give the opportunity to extend this study to dense materials, where the equilibration time is long, such as glasses and melts of geological interest, actinide oxides fuels, or irradiated metallic alloys.

\section{ASSOCIATED CONTENT}

\section{S Supporting Information}

Additional data showing the $\mathrm{He}$ and $\mathrm{Ne}$ adsorbed amounts as a function of pressure for different strains. This material is available free of charge via the Internet at http://pubs.acs.org.

\section{AUTHOR INFORMATION}

\section{Corresponding Authors}

*E-mail: coasne@mit.edu (B.C.).

*E-mail: benoit.ruffle@univ-montp2.fr (B.R.).

\section{Notes}

The authors declare no competing financial interest.

\section{ACKNOWLEDGMENTS}

The authors thank Eric Courtens for his critical reading of the manuscript, Sébastien Clément and Rémy Vialla for their help during the experiments, and Christelle Dupas for the sample preparation. The work was partially supported by Région Languedoc-Roussillon (Omega Platform).

\section{REFERENCES}

(1) Shelby, J. Helium Migration in Natural and Synthetic Vitreous Silica. J. Am. Ceram. Soc. 1972, 55, 61-64.

(2) Shelby, J. Pressure-Dependence of Helium and Neon Solubility in Vitreous Silica. J. Appl. Phys. 1976, 47, 135-139.

(3) Shackelford, J.; Masaryk, J. Interstitial Structure of Vitreous Silica. J. Non-Cryst. Solids 1978, 30, 127-134.

(4) Chan, S.-L.; Elliott, S.-R. Theoretical Study of the Interstice Statistics of the Oxygen Sublattice in Vitreous $\mathrm{SiO}_{2}$. Phys. Rev. B 1991, 43, 4423-4432.

(5) Sato, T.; Funamori, N.; Yagi, T. Helium Penetrates into Silica Glass and Reduces Its Compressibility. Nat. Commun. 2011, 2, 345.

(6) Shen, G.; Mei, Q.; Prakapenka, V.-B.; Lazor, P.; Sinogeikin, S.; Meng, Y.; Park, C. Effect of Helium on Structure and Compression Behavior of $\mathrm{SiO}_{2}$ Glass. Proc. Natl. Acad. Sci. U.S.A. 2011, 108, 60046007.

(7) Weigel, C.; Polian, A.; Kint, M.; Rufflé, B.; Foret, M.; Vacher, R. Vitreous Silica Distends in Helium Gas: Acoustic Versus Static Compressibilities. Phys. Rev. Lett. 2012, 109, 245504.

(8) Klotz, S.; Chervin, J.-C.; Munsch, P.; Le Marchand, C. Hydrostatic Limits of 11 Pressure Transmitting Media. J. Phys. D: Appl. Phys. 2009, 42, 075413.

(9) Kondo, K.; Lio, S.; Sawaoka, A. Non-Linear Pressure-Dependence of the Elastic Moduli of Fused Quartz up to 3 GPa. J. Appl. Phys. 1981, 52, 2826-2831.

(10) Schroeder, J.; Bilodeau, T.-G.; Zhao, X.-S. Brillouin and Raman Scattering from Glasses under Pressure. High Pressure Res. 1990, 4, 531-533.

(11) Zha, C.-S.; Hemley, R.-J.; Mao, H.-K.; Duffy, T.-S.; Meade, C. Acoustic Velocities and Refractive Index of $\mathrm{SiO}_{2}$ Glass to 57.5 GPa by Brillouin Scattering. Phys. Rev. B 1994, 50, 13105-13112.

(12) Meade, C.; Jeanloz, R. Frequency Dependent Equation of State of Fused Silica up to 10 GPa. Phys. Rev. B 1987, 35, 236-244.

(13) Tsiok, O.; Brazhkin, V.; Lyapin, A.; Khvostantsev, L. Logarithmic Kinetics of the Amorphous-Amorphous Transformations in $\mathrm{SiO}_{2}$ and $\mathrm{GeO}_{2}$ Glasses under High-Pressure. Phys. Rev. Lett. 1998, 80, 999-1002.

(14) Coussy, O. Mechanics and Physics of Porous Solids; John Wiley \& Sons, Ltd: Chichester, U.K., 2010.

(15) Serre, C.; Mellot-Draznieks, C.; Surble, S.; Audebrand, N.; Filinchuk, Y.; Ferey, G. Role of Solvent-Host Interactions that Lead to Very Large Swelling of Hybrid Frameworks. Science 2007, 315, $1828-1831$

(16) Coudert, F.-X.; Jeffroy, M.; Fuchs, A. H.; Boutin, A.; MellotDraznieks, C. Thermodynamics of Guest-Induced Structural Transitions in Hybrid Organic-Inorganic Frameworks. J. Am. Chem. Soc. 2008, 130, 14294-14302.

(17) Günther, G.; Prass, J.; Paris, O.; Schoen, M. Novel Insights into Nanopore Deformation Caused by Capillary Condensation. Phys. Rev. Lett. 2008, 101, 086104.

(18) Neimark, A. V.; Coudert, F.-X.; Boutin, A.; Fuchs, A. H. StressBased Model for the Breathing of Metal-Organic Frameworks. J. Phys. Chem. Lett. 2010, 1, 445-449.

(19) Long, Y.; Palmer, J. C.; Coasne, B.; Sliwinska-Bartkowiak, M.; Gubbins, K. E. Pressure Enhancement in Carbon Nanopores: A Major Confinement Effect. Phys. Chem. Chem. Phys. 2011, 13, 17163-17170.

(20) Carmeliet, J.; Derome, D.; Dressler, M.; Guyer, R. A. Nonlinear Poro-Elastic Model for Unsaturated Porous Solids. J. Appl. Mech. 2013, 80, 020909.

(21) Brochard, L.; Vandamme, M.; Pellenq, R. J.-M. Poromechanics of Microporous Media. J. Mech. Phys. Solids 2012, 60, 606-622.

(22) Ayrinhac, S.; Rufflé, B.; Foret, M.; Tran, H.; Clement, S.; Vialla, R.; Vacher, R.; Chervin, J. C.; Munsch, P.; Polian, A. Dynamical Origin of Anomalous Temperature Hardening of Elastic Modulus in Vitreous Silica. Phys. Rev. B 2011, 84, 024201.

(23) Vacher, R.; Ayrinhac, S.; Foret, M.; Rufflé, B.; Courtens, E. Finite Size Effects in Brillouin Scattering from Silica Glass. Phys. Rev. B 2006, 74, 012203. 
(24) Whitfield, C.; Brody, E.; Basset, W. Elastic Moduli of $\mathrm{NaCl}$ by Brillouin Scattering at High-Pressure in a Diamond Anvil Cell. Rev. Sci. Instrum. 1976, 47, 942-947.

(25) Biot, M. A. General Theory of Three-Dimensional Consolidation. J. Appl. Phys. 1941, 12, 155-164.

(26) Gor, G. Y.; Neimark, A. V. Adsorption-Induced Deformation of Mesoporous Solids. Langmuir 2010, 26, 13021-13027.

(27) Espinoza, D.; Vandamme, M.; Dangla, P.; Pereira, J.; VidalGilbert, S. A Transverse Isotropic Model for Microporous Solids: Application to Coal Matrix Adsorption and Swelling. J. Geophys. Res.: Solid Earth 2013, 118, 6113-6123.

(28) Mantisi, B.; Tanguy, A.; Kermouche, G.; Barthel, E. Atomistic Response of a Model Silica Glass under Shear and Pressure. Eur. Phys. J. B 2012, 85, 304.

(29) Coasne, B.; Haines, J.; Levelut, C.; Cambon, O.; Santoro, M.; Gorelli, F.; Garbarino, G. Enhanced Mechanical Strength of Zeolites by Adsorption of Guest Molecules. Phys. Chem. Chem. Phys. 2011, 13, 20096-20099.

(30) Sarkisov, L.; Monson, P. A. Hysteresis in Monte Carlo and Molecular Dynamics Simulations of Adsorption in Porous Materials. Langmuir 2000, 16, 9857-9860.

(31) Carré, A.; Horbach, J.; Ispas, S.; Kob, W. New Fitting Scheme to Obtain Effective Potential from Car-Parrinello Molecular-Dynamics Simulations: Application to Silica. Europhys. Lett. 2008, 82, 17001.

(32) Lee, S.; Rossky, P. A Comparison of the Structure and Dynamics of Liquid Water at Hydrophobic and Hydrophilic Surfaces - A Molecular Dynamics Simulation Study. J. Chem. Phys. 1994, 100, 3334-3345.

(33) Hirschfelder, J. O., Curtiss, C. F., Bird, R. B., Eds. Molecular Theory of Gases and Liquids; Wiley Interscience: New York, 1954; $\mathrm{p}$ 1114.

(34) Poling, B. E., Prausnitz, J. M., O'Connell, J. P., Eds. The Properties of Gases and Liquids, 5th ed.; McGraw-Hill: New York, 2001.

(35) Letoullec, R.; Loubeyre, P.; Pinceaux, J. Refractive-Index Measurements of Dense Helium up to $16 \mathrm{GPa}$ at $\mathrm{T}=298 \mathrm{~K}-$ Analysis of Its Thermodynamics and Electronic-Properties. Phys. Rev. B 1989, 40, 2368-2378.

(36) Dewaele, A.; Eggert, J.; Loubeyre, P.; Le Toullec, R. Measurement of Refractive Index and Equation of State in Dense $\mathrm{He}, \mathrm{H}_{2}, \mathrm{H}_{2} \mathrm{O}$, and Ne under High Pressure in a Diamond Anvil Cell. Phys. Rev. B 2003, 67, 094112.

(37) Efimchenko, V. S.; Fedotov, V. K.; Kuzovnikov, M. A.; Zhuravlev, A. S.; Bulychev, B. M. Hydrogen Solubility in Amorphous Silica at Pressures up to 75 kbar. J. Phys. Chem. B 2013, 117, 422-425.

(38) Huang, L.; Kieffer, J. Structural Origin of Negative Thermal Expansion in High-Temperature Silica Polymorphs. Phys. Rev. Lett. 2005, 95, 215901.

(39) Walker, A. M.; Sullivan, L. A.; Trachenko, K.; Bruin, R. P.; White, T. O. H.; Dove, M. T.; Tyer, R. P.; Todorov, I. T.; Wells, S. A. The Origin of the Compressibility Anomaly in Amorphous Silica: A Molecular Dynamics Study. J. Phys.: Condens. Matter 2007, 19, 275210.

(40) Guillot, B.; Sator, N. Noble Gases in High-Pressure Silicate Liquids: A Computer Simulation Study. Geochim. Cosmochim. Acta 2012, 80, 51-69.

(41) Boutéca, M. J.; Guéguen, Y. Mechanical Properties of Rocks: Pore Pressure and Scale Effects. Oil and Gas Science and Technology Rev. IFP 1999, 54, 703-714.

(42) Rice, J. R.; Cleary, M. P. Some Basic Stress-Diffusion Solutions for Fluid Saturated Elastic Porous Media with Compressible Constituents. Rev. Geophys. Space Phys. 1976, 14, 227-241.

(43) Sarda, P.; Guillot, B. Breaking of Henry's Law for Noble Gas and $\mathrm{CO}_{2}$ Solubility in Silicate Melt under Pressure. Nature 2005, 436, 95-98. 